\title{
Intra-operative computed-tomography-like real-time three-dimensional ultrasound in neurosurgery
}

\author{
Bozinov, O ; Burkhardt, J K
}

\begin{abstract}
Intra-operative ultrasound (ioUS) is one alternative for neurosurgeons to obtain images during surgery. Inexpensive to purchase and maintain, as well as convenient and easy to use, the functionality of ioUS is wide in scope. Many different US probes with various characteristics are available to localize and visualize intracerebral lesions, as well as to serve as a resection control during surgery. These probes include frequency range, matrix phase arrays, and imaging mode $(2 \mathrm{D}, 3 \mathrm{D}, 4 \mathrm{D})$ - many with additional features such as navigation or color duplex, depending on the aim of use. Many neurosurgeons, however, avoid ioUS because of some resolution and orientation problems, or simply because of inexperience.
\end{abstract}

DOI: https://doi.org/10.1016/j.wneu.2012.05.025

Posted at the Zurich Open Repository and Archive, University of Zurich ZORA URL: https://doi.org/10.5167/uzh-72830

Journal Article

Accepted Version

Originally published at:

Bozinov, O; Burkhardt, J K (2012). Intra-operative computed-tomography-like real-time three-dimensional ultrasound in neurosurgery. World Neurosurgery, 78(1-2):5-7.

DOI: https://doi.org/10.1016/j.wneu.2012.05.025 


\section{Intra-operative CT-like 3D Ultrasound in Neurosurgery}

Oliver Bozinov and Jan-Karl Burkhardt

Intra-operative ultrasound (ioUS) became firmly established in neurosurgery within the last decades. Both low initial and maintenance costs and its ease of use led to its widely application as an intra-operative imaging tool. Many different ultrasound probes with various characteristics are available to localize and visualize intracerebral lesions, as well as to serve as a resection control during surgery. For instances, frequency range, matrix phase arrays, imaging mode (2D, 3D, 4D) or additional features including navigation or color duplex differ in the available ioUS probes depending on the aim of use.

The recently introduced 3D ioUS probes in neurosurgery are of special interest, since intracerebral lesion can now be displayed in familiar planes (axial, coronal and sagittal) during surgery. In other medical subspecialities 3D US is already established as a diagnostic tool. For instances, in cardiology 3D US became essential to evaluate ventricular volumes of the heart. (Séguéla PE et al. "Feasibility of Three-Dimensional Transthoracic Echocardiography to Evaluate Right Ventricular Volumes in Children and Comparison to Left Ventricular Values." Echocardiography. 2011 Dec 9. doi: 10.1111/j.15408175.2011.01596.x. [Epub ahead of print]).

Although prospective studies with 3D ioUS are still missing in neurosurgery, first results showed its promising potential as an intraoperative diagnostic tool as well as a surgical resection aid especially in tumor surgery. For instances, in our recently published study we showed that $3 \mathrm{D}$ ioUS was useful to localize various pediatric brain tumors and was helpful to assist the resection of these lesions in live two-plane imaging before and after dura opening. (Ulrich NH et al. "Resection of pediatric intracerebral tumors with the aid of intraoperative real-time 3-D ultrasound.” Childs Nerv Syst. 2011 Sep 17. [Epub ahead of print]). Although the range of ultrasound coverage was limited due to the US probe (X7-2, 
Philips), superficial as well as deep-seated tumors located in the brain hemisphere of the surgical approach could be displayed. However, a view to the other side of the hemisphere up to the opposite skull was limited to the size of matrix elements $(2,500)$ leading to sound shadows and artifacts in wider sound ranges.

A novel ioUS probe (X6-1, Philips) with a larger scale of 9,212 matrix elements was recently tested at our department to overcome this limitation. Although the size of the US probe is larger than previous US probes we were able to use this probe as an intra-operative CT like imaging tool of the whole brain. After craniotomy with opened or closed dura the probe was able to sound through the whole brain parenchyma to the opposite skull providing a complete overview of the brain. Using the 3D mode, the brain could be imaged real-time in axial, coronal and sagittal planes (Figure 1a) and this probe provides the neurosurgeon with novel valuable information during surgery. For instances, in neuro-traumatology this US probe might be useful to detect intracerebral lesion such as an epidural or subdural hematoma of the contralateral side during surgery. Also in patients received a craniectomy this probe might be suitable for bedside follow-up scans detecting a midline shift or to measure the size of the ventricles. However, a pitfall of the appliance of this probe is that an intracerebral lesion might still mask the view behind the lesion with its sound shadow and therefore leads to an impaired interpretation of this brain area. An other interesting feature of this probe consists of an ultrasound-based angiography without using contrast, as seen by the arterial vessel reconstruction of the Circle of Willis (Figure 1b). Although the main supplying arteries of the brain could be detected, the imaging quality of the angiography is still not satisfactory enough to accurately specify the detailed structure of the main vessels or display smaller branches. However, the results are promising and the imaging quality will hopefully improve in future with novel probes.

In summary, ioUS technology in neurosurgery is still in motion and novel technical improvements lead to further promising applications during surgery. With the novel CT like 
ioUS imaging we are now able to get a fast and detailed overview of both brain hemispheres during surgery without using conventional CT or MR imaging.

Fig 1 

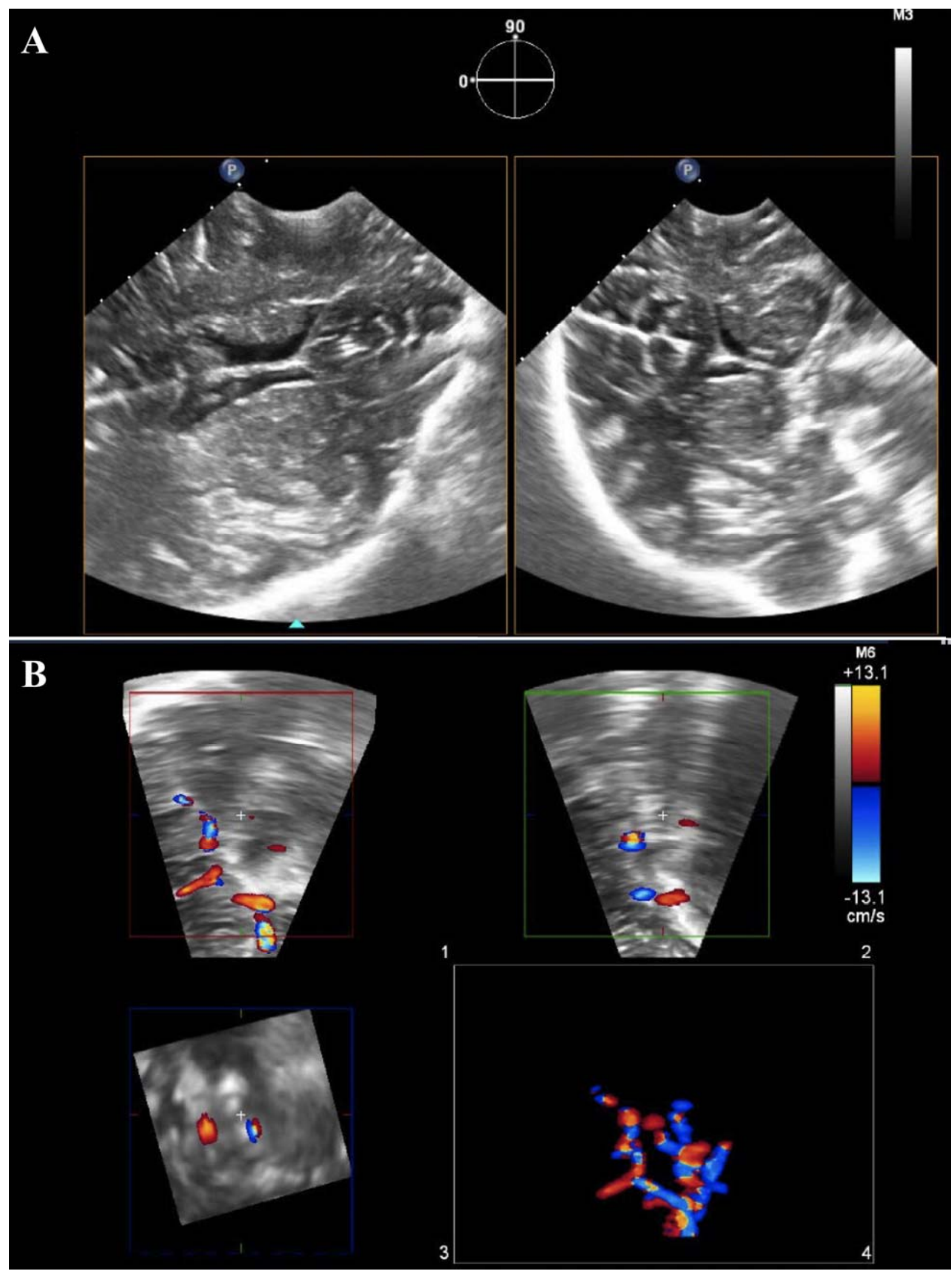

Fig 2 


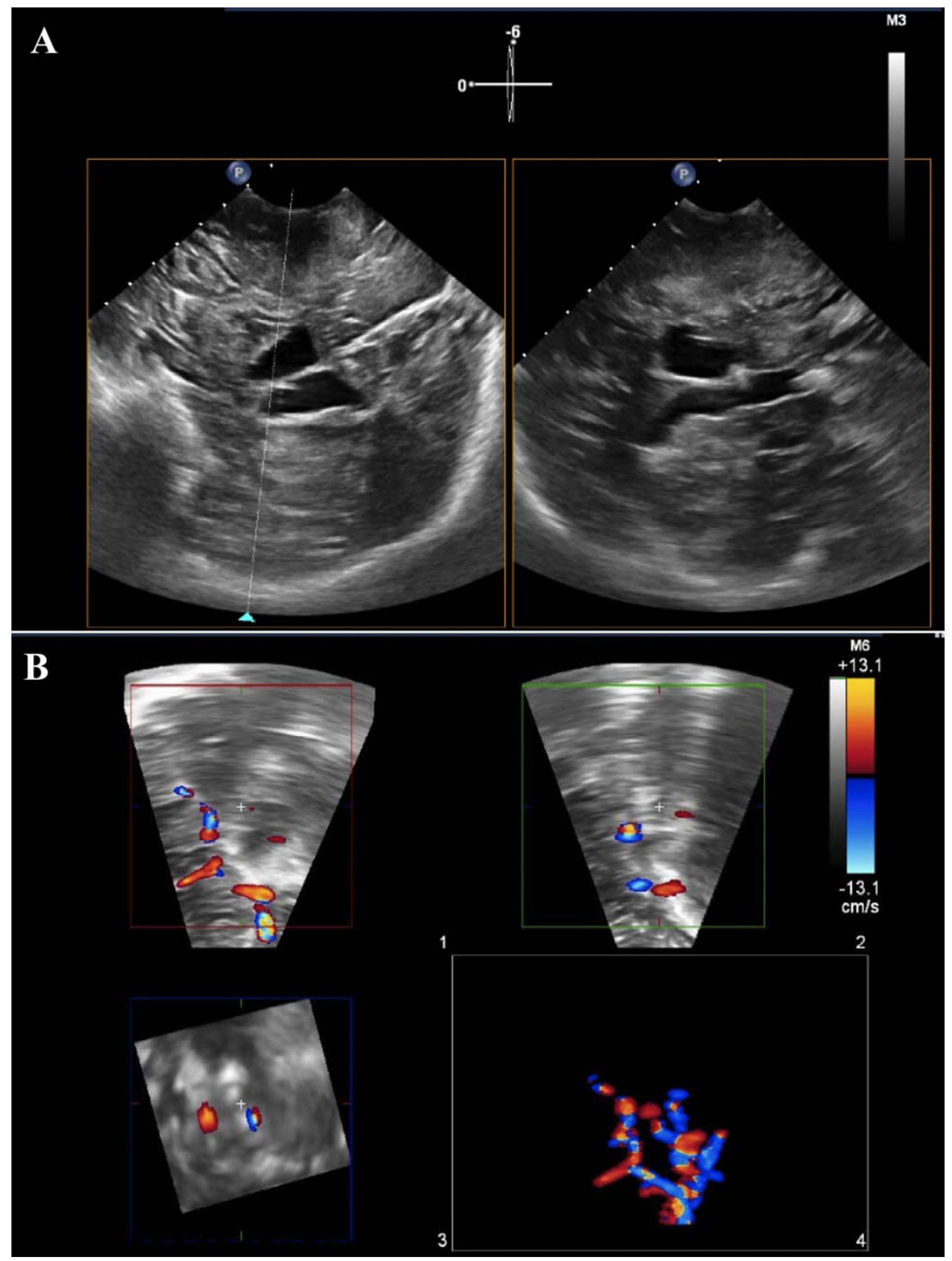

